Background Trans-radial access for neuro-endovascular procedures has gained popularity in recent years, driven in part by social media. The approach was adopted and modified from that used by interventional cardiology. This study analyzes Twitter conversations regarding this topic using the popular hashtag \#RadialFirst.

Materials and Methods Twitter was evaluated for public content pertaining to all trans-radial access. We conducted a mixed-method analysis that includes a quantitative examination of all social media metrics (no. of tweets, mentions, retweets, locations, word count) and a qualitative evaluation of tweets for thematic analysis.

Results Between January 2013 and February 2020, there were 100,531 tweets from 100 countries using the hashtag \#RadialFirst. These tweets had an overall impression of 218 million and were retweeted 75,000 times by 13,103 users. More than $60 \%$ of tweets carried media materials (e.g. wrist image, angiographic runs) but only $14 \%$ of tweets had a link to an article. USA (17\%) and the UK (4.2\%) had the highest numbers of tweets. Physicians authored $84 \%$ of the tweets and interventional cardiologists accounted for 94\% of the top 100 influencers for the hashtag. Tweets showed steady increase in numbers over time and coincided later with the appearance of a new hashtag for neuro-intervention, \#RadialForNeuro. Five prominent themes emerged from the coded data: sharing cases, providing operative tips, requesting information,

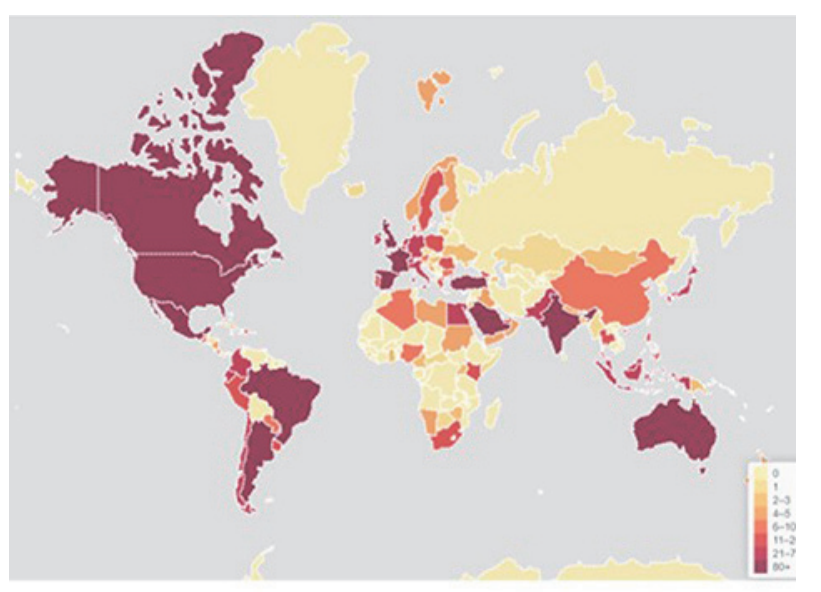

World map

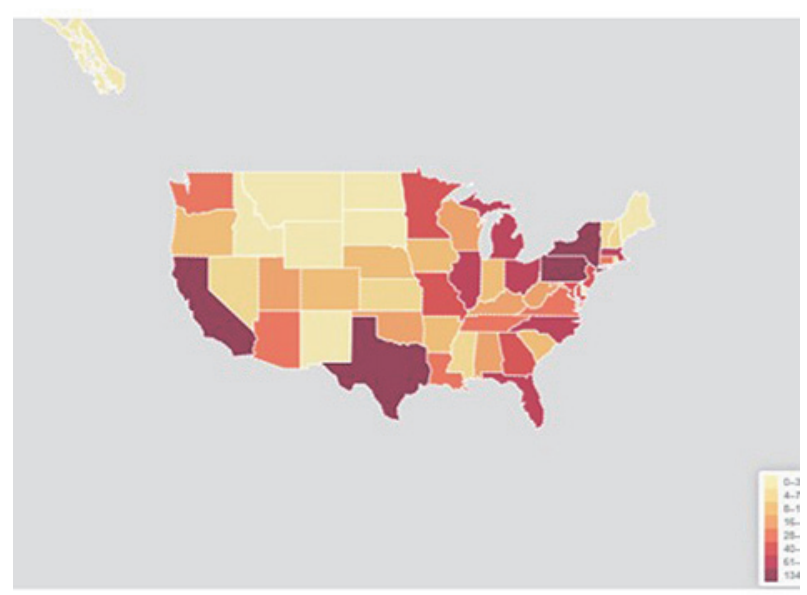

US map highlighting adverse events, and tweets related to commercial endorsements.

Conclusion This study is the first to provide insight into Twitter communications regarding trans-radial access. These findings highlight the impact of Twitter in disseminating the technique, and the role of social media in education and refinement of technical approaches.

Disclosures N. Alotaibi: None. P. Harker: None. F. Robertson: None. J. Vranic: None. M. Koch: None. C. Stapleton: None. T. Leslie-Mazwi: None. A. Patel: None.

\section{E-084 VARIABILITY IN ENDOVASCULAR TREATMENT OF INTRACRANIAL INTERNAL CAROTID ARTERY VERSUS MIDDLE CEREBRAL ARTERY OCCLUSIONS}

M Aly*, R Abdalla, M Hurley, A Shaibani, S Ansari. Intervention Radiology, Northwestern University, Chicago, IL

\subsection{6/neurintsurg-2020-SNIS. 118}

Purpose Endovascular treatment (ET) for the acute management of ischemic stroke due to large vessel occlusions is the standard of care for anterior circulation. Meta-analysis has suggested equivalent recanalization and better clinical outcomes after ET for ICA and proximal MCA occlusion (1-2). The aim of our study was to compare these two cohorts and identify any variability/bias in presentations, imaging selection, technical and clinical efficacy.

Materials and Methods This is a retrospective study of patients that underwent ET for ICA/MCA occlusions over a four-year period at three comprehensive stroke centers. We studied patient demographics, vascular risk factors, NIHSS, time of presentation, imaging, procedures details, and clinical outcomes. Chi-square, Mann-Whitney $U$ and student ttests were used for variables univariate analysis as appropriate. Multivariate analysis was performed to assess the comparability of the groups based on demographics and risk factors.

Results 185 patients were studied (mean age, $68 \pm 17$; 55\% women; median NIHSS, $16 \pm 6$ ). Both study cohorts were comparable regarding their age $(65 \pm 20$ vs $69 \pm 17$, $\mathrm{p}=0.26)$ and past medical history of Hypertension, Diabetes Mellitus, Hyperlipidemia and Smoking habit $(\mathrm{p}=0.64$, $\mathrm{p}=0.68, \mathrm{p}=0.55, \mathrm{p}=0.47$ respectively). There was no statistically significant difference between both cohorts regarding their NIHSS $(17 \pm 6$ vs $16 \pm 6, p=0.23)$ or the time from onset to groin puncture (mean 5:43:48 vs 5:24:58, $\mathrm{p}=0.54)$. Moreover, there was no significant difference between mean ASPECT $(8.3 \pm 1.3$ vs $8.6 \pm 1.3, \mathrm{p}=0.19)$ and mean CTP-rCBF $<30 \%(24.5 \mathrm{ml} \pm 36$ vs $28.3 \mathrm{ml} \pm$ $38, \mathrm{p}=0.6)$. During the procedure ICA occlusions were associated with a higher number of stent retriever passes to achieve recanalization (mean 2.5 vs $1.9, \mathrm{p}=0.028$ ), and longer time from groin puncture to recanalization (mean 1:02:11 vs 00:51:48, $\mathrm{p}=0.034)$. Also, there was a trend toward significance in the rate of successful recanalization after the first pass of stent retriever in the MCA occlusion cohort $(46 \%$ vs $33 \%, \mathrm{p}=0.09)$. However, there was no statistically significant difference regarding the rate of successful recanalization TICI $\geq 2$ b $(91 \%$ in group A vs $95 \%$ in group $\mathrm{B}, \mathrm{p}=0.35)$ or the rate of complete recanalization TICI $2 \mathrm{c} / 3$ (45\% vs $52 \%, \mathrm{p}=0.39)$. Although, the 90 -days outcome measured by mRS was not statistically significantly different 
between both groups (mean 2.5 vs $3.1, \mathrm{p}=0.09$ ), however, the outcome was trending to be worse in MCA occlusion patients, this could be the result of the relatively higher rate of procedural complications in MCA occlusions, $18.5 \%$ in MCA (24/130 cases, 13 distal embolic, $8 \mathrm{SAH} / \mathrm{sICH}, 3$ arterial dissections) versus $12.5 \% \quad(7 / 55$ cases, 4 distal embolic, $3 \mathrm{SAH} / \mathrm{sICH})$.

Conclusions Significant procedural difficulty is encountered during ET of ICA relative to MCA occlusions, requiring increased procedural time and passes. Although final successful recanalization rates are equivalent, we noted a paradoxical trend for less functional independence with MCA occlusions this could be due to the higher rate of complications in the MCA cohort, and the better collaterals through the circle of Willis in the ICA cohort.

Disclosures M. Aly: None. R. Abdalla: None. M. Hurley: None. A. Shaibani: None. S. Ansari: None.

\section{E-085 MULTICENTER RETROSPECTIVE STUDY OF THE SOFIA FOR ASPIRATION IN ACUTE ISCHEMIC STROKE (MRS SOFIA)}

${ }^{1} \mathrm{~W}$ Brinjikji*, ${ }^{2} \mathrm{R}$ Deleacy, ${ }^{3} \mathrm{D}$ Meila, ${ }^{4} \mathrm{M}$ Mokin, ${ }^{5} \mathrm{E}$ Samaniego, ${ }^{6} \mathrm{~A}$ Cora, ${ }^{7} \mathrm{M}$ Shapiro, ${ }^{7} \mathrm{~S}$ Agarwal, ${ }^{7} \mathrm{D}$ Bageac, ${ }^{5} \mathrm{~J}$ Roa, ${ }^{4} \mathrm{Z}$ Ren, ${ }^{4} \mathrm{~W} \mathrm{Li},{ }^{8} \mathrm{~L}$ Rinaldo, ${ }^{1} \mathrm{H}$ Cloft, ${ }^{6} \mathrm{D}$ Volders, ${ }^{6} \mathrm{~T}$ Huynh, ${ }^{7}$ E Raz. ${ }^{1}$ Radiology, Mayo Clinic, Rochester, MN; ${ }^{2}$ Radiology, Mt. Sinai, New York, NY; ${ }^{3}$ Radiology, Hannover Medical Center, Hannover, GERMANY; ${ }^{4}$ Neurology, University of South Florida, Tampa, FL; ${ }^{5}$ Neurology, University of lowa, Hawkins City, IA; ${ }^{6}$ Radiology, Dalhousie, Halifax, NS, CANADA; 'Radiology, NYU Medical Center, New York, NY; ${ }^{8}$ Neurosurgery, Mayo Clinic, Rochester, MN

\subsection{6/neurintsurg-2020-SNIS.119}

Background and Purpose There are a myriad of aspiration catheters used for primary aspiration for mechanical embolectomy for acute ischemic stroke. Over the past several years there has been increased interest in the use of the 6Fr Sofia Plus aspiration catheter as a primary aspiration catheter due to its trackability and navigability. We performed a multicenter retrospective study examining the efficacy of the Sofia aspiration catheter as a stand-alone aspiration treatment for large vessel occlusion.

Materials and Methods This was an investigator initiated study and no study funding was received. Following Institutional Review Board Approval, consecutive cases in which the Sofia catheter was used for aspiration thrombectomy for treatment of large vessel occlusion were included. Exclusion criteria were the following: 1) Sofia not used for first pass, and 2) stent-triever used as an adjunct on the first pass. The primary outcome of the study was first pass recanalization (TICI 2b/3). Secondary outcomes included first pass TICI $2 \mathrm{c} / 3$, final TICI using Sofia alone, cross-over to other thrombectomy devices, number of passes, time from puncture to recanalization and complications.

Results From the six centers, a total of 323 patients were included. First pass recanalization with TICI 2b/3 was achieved in $69.7 \%$ of cases. First pass recanalization with TICI $2 \mathrm{c} / 3$ was achieved in $48.9 \%$ of cases. $74.8 \%$ patients achieved a TICI $2 \mathrm{~b} / 3$ with the Sofia alone. Cross-over to other thrombectomy devices occurred in $29.1 \%$ of cases. The median number of passes performed was 1.5 (IQR=1-3). Median time from puncture to recanalization was 25 $(\mathrm{IQR}=15-35)$. Procedure related complications occurred in $2.8 \%$ of cases.
Conclusion Our study highlights the potential advantage of the $5 \mathrm{Fr}$ and $6 \mathrm{Fr}$ Sofia aspiration catheters for primary aspiration thrombectomy in acute ischemic stroke. We were able to achieve high rates of first pass recanalization with low cross-over rates to other thrombectomy devices. Median procedure time was low as were procedural complications.

Disclosures W. Brinjikji: 1; C; NIH/NINDS, Cerenovus. 2; C; Microvention, Cerenovus. 4; C; Marblehead Medical LLC. R. DeLeacy: None. D. Meila: None. M. Mokin: None. E. Samaniego: None. A. Cora: None. M. Shapiro: None. S. Agarwal: None. D. Bageac: None. J. Roa: None. Z. Ren: None. W. Li: None. L. Rinaldo: None. H. Cloft: None. D. Volders: None. T. Huynh: None. E. Raz: None.

\section{E-087 SAFETY AND EFFICACY OF TRACSTAR LARGE DISTAL PLATFORM DURING MECHANICAL THROMBECTOMY}

D Bageac*, R De Leacy. Neurosurgery, The Mount Sinai Hospital, New York, NY

\subsection{6/neurintsurg-2020-SNIS.120}

Introduction Mechanical thrombectomy is the treatment of choice for ischemic stroke due to large vessel occlusion. One common reason for failure or prolonged procedure time is vascular touristy inhibiting access to the site of occlusion. A novel device -the TracStar Large Distal Platform (LDP) -may offer improved navigation of tortuous vasculature as well as more distal final positioning when compared to commonly used guide catheters. We investigate the safety and efficacy of the TracStar LDP when used during mechanical thrombectomy.

Methods We perform a multicenter retrospective review of mechanical thrombectomy procedures during which the TracStar LDP was utilized. Procedural information, complications, and key timepoints (groin puncture, clot-first-touch, etc.) were recorded as detailed in the operative note. Vascular tortuosity was assessed via pre-procedural CTA. Distal-most position achieved with TracStar was determined by review of intraprocedural imaging. Linear regression was performed to investigate the potential relationship between vascular tortuosity and procedure time.

Results In a preliminary analysis of 20 cases, successful reperfusion (TICI $2 \mathrm{~b} / 3$ ) was achieved in $88 \%$ of cases, requiring 1.8 passes on average (rage 1-5). Average NIHSS at presentation was $17.8 \pm 6$. Anterior circulation emboli were encountered in 15 cases. Average time to clot-first touch was $12 \pm 7$ minutes and average time to final reperfusion was $50 \pm 27.6$ minutes. Good functional outcome (mRS 0-2) at 90 days was achieved in $35 \%$ of cases. There was no association between vascular tortuosity and procedure time. There were no procedural complications, and TracStar LDP was exchanged for an alternative guide catheter in two cases due to failure to maintain positing.

Conclusion Mechanical thrombectomy with the Tracstar LDP is safe and effective. Access beyond the horizonal petrous segment of the ICA was achieved in the majority of cases. Interestingly, ICA tortuosity was not associated with an increased time to clot-first-touched. Final analysis of 30 patients in this multi-center retrospective study will be available for presentation at the SNIS Annual Meeting 2020 .

Disclosures D. Bageac: None. R. De Leacy: None. 